\title{
Interactive braille output for blind computer users
}

\author{
TERRENCE T. WILLIAMS, ROBERT M. LAMBERT, and CHARLES W. WHITE \\ Concordia University, Montreal, Quebec, Canada
}

\begin{abstract}
Blind readers were tested using two methods of reading text displayed by an Apple microcomputer. The first method employed an Optacon system, a device that displays tactile representations of single characters, and the second used an interactive single electronic braille cell that displayed grade 1 braille characters. The results demonstrated no difference in accuracy or reading speed between these two methods. Thus, the serial presentation of braille characters at a single position appears to be a viable method of information transfer between computers and braille readers.
\end{abstract}

Braille writing for the blind was developed by Louis Braille in 1824. It is a method of encoding individual characters and phrases into a format legible by the blind or visually impaired. The technique uses a $2 \times 3$ dot matrix to represent individual characters and commonly used words. The braille matrix is typically formed on paper by embossing raised dots; in this way, the braille reader is able to recognize characters by touch. This method of encoding the written word for the blind is described by many as the preferred method of information transfer. Although other transcription techniques are available, reading rates with these are slower than with braille.

The spoken word, presented with current cassette recording techniques, is an alternative to braille text, but listening rates are low and the user must depend on second-party assistance to record the text. The latter problem can be avoided when the speech is synthesized by computer. In some cases, this method has been found to be useful in presenting material stored in computer memory.

A third technique, in addition to braille and the spoken word, is reading printed text with the aid of the Optacon (Telesensor Systems, 1971a). This device presents the user with a vibrotactile array of $6 \times 24$ individual points that correspond to a photosensitive array upon which text is imaged. The hand-held camera used to view text is capable of imaging either printed text or characters displayed on a computer's video monitor. The user is able to read text with the Optacon by positioning the camera and then reading by touch the character generated on the vibrotactile display. The information used by the reader is based upon two-dimensional contours; the characters read by this device are not encoded in any way, but can be transformed by means of optical magnification.

The authors' mailing address is: Department of Psychology, Concordia University, 7141 Sherbrooke Street West, Montreal, Quebec, Canada H4B 1R6.
These methods adopted by the blind and visually impaired were originally designed to be used with printed text or as alternatives to printed text; the communication medium they were invented to cope with was the printed word. Other problems arise when these techniques are adapted for use with a similar yet distinct medium, computer-generated text. The difficulties arise primarily in the area of the interaction between the blind user and the computer and not specifically in the method used to display information. Two popular methods are the use of the Optacon to read computer monitor displays and the use of paperless braille display units such as the Versabraille (Telesensory Systems, 1971b) and Microbrailler (Triformation Systems, 1971) that can accept ASCII-coded output. A distinct advantage of the Optacon system is that it is independent of hardware and software; a disadvantage is the necessity of interpreting shapes displayed on the vibrotactile matrix. The paperless braille displays encode information into a familiar braille format that is typically read without difficulty and with certainty by braille readers; however, these units are not transparent to the user. That is, software must be configured to allow the use of these devices or to have an option incorporated that provides for a remote terminal. In most cases, software designed for personal computers does not include these options.

The advantages to be found in each of these approaches are the ease of reading the braille display in the paperless braille units and the ease of use of the Optacon with software not especially configured for the blind. In an attempt to satisfy the requirements of the blind computer user and to optimize the interaction between user and computer, an interactive braille display unit that attempted to incorporate these advantages was designed. To evaluate the design, a comparison of performance was made with the new device and an Optacon system. The Optacon was selected because it is readily adaptable for computer use and it employs a serial method of character presentation. The new design is similar to the Optacon in that it is sim- 
ple to operate and also uses a serial presentation of characters at a fixed spatial location; a significant difference is the use of braille characters on a single braille cell rather than character contours on a vibrotactile display.

\section{SUBJECTS}

Two blind braille readers participated in the experiment. In both cases, reading rates using conventional embossed braille characters on paper ranged between 8 and 12 characters per second. Both readers had at least 2 years of daily experience with the Optacon.

\section{APPARATUS}

A microcomputer (Apple //e) with a green phosphor video monitor (Apple //I) was used in both conditions. An Optacon print reading system with an adjustable CRT lens module was used in a free viewing manner without aids to assist horizontal or vertical tracking on the screen. A single dynamic braille cell was constructed to display grade 1 braille characters. The single cell allowed virtually simultaneous updating of individual points on the $2 \times 3$ matrix. Vertical spacing of the individual points was set at $2.5 \mathrm{~mm}$, and horizontal spacing was $2 \mathrm{~mm}$, measured from center to center. Individual pins in the cell had a diameter of $1.25 \mathrm{~mm}$. The overall cell size was $4 \mathrm{~mm}$ wide and $7 \mathrm{~mm}$ long. The individual pins were actuated using an electromechanical device, and each pin was controlled individually. The maximum rate of presentation of individual braille characters with the standard autorepeat function was 16 characters/sec. Rate of presentation was controlled by the user. A schematic representation of the output devices used in each condition is presented in Figure 1.

Software developed for this application allowed the user to select any character position on the computer's $\mathbf{8 0}$ column $\times 24$ row display by using arrow direction keys on the keyboard (see Figure 2). The alphanumeric character in the selected position was read, translated to braille,

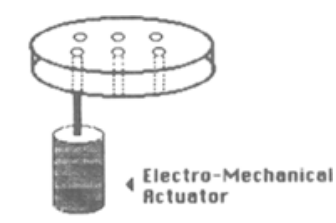

Single - Cell Dynamic Braille Display

Figure 1. An illustration of the tactile arrays used in each condition.

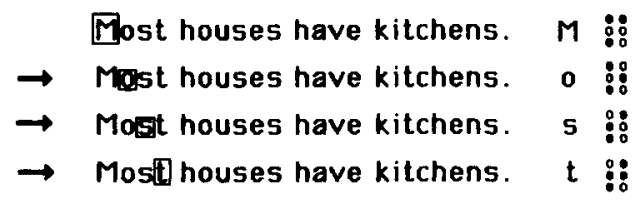

Figure 2. The method used to update the single cell braille display employed single keystrokes to change the position of the character displayed.

and then displayed at the dynamic braille cell. As the selected position was changed using keyboard input, the dynamic braille cell was immediately updated; in this way, the user could change character position with one hand while reading braille output with the other. The dynamic braille cell was interfaced with the microcomputer by using a 6522 versatile interface adapter.

The text used to measure reading rates was constructed of independent sentences describing simple common events that could easily be labeled as true or false. The same sentences were used in both conditions, but the order of presentation was determined randomly for each condition.

\section{PROCEDURE}

Subjects were instructed to read text aloud and to describe each statement as true or false at the end of each sentence. For each condition, 42 statements were presented randomly in groups of 6 , and voice recordings were used after each session to determine reading rates. Using the Optacon in one condition, subjects were given the opportunity to practice by reading a group of statements on the CRT at the beginning of the test session. The readers were instructed that the first statement would always appear in the upper left-hand corner of the monitor and that the other statements would be positioned immediately below the first. The same procedure was used during the braille reading session, with the addition of instructions describing the operation of the braille device. These instructions included the use of arrow keys at the keyboard to change the braille character displayed, the return key to move to the beginning of the next line, and key " $\mathrm{H}$ " (for "home" position) to return to the first position of the top line displayed on the monitor. In total, the practice session with each device lasted a maximum of $15 \mathrm{~min}$. This practice period was the only experience provided with the dynamic braille display unit before testing.

In both conditions, the reader was able to control text output by either moving the Optacon camera to update the vibrotactile display or by using the cursor-control keys to change the single character displayed at the braille cell.

\section{RESULTS AND DISCUSSION}

The measure of performance with each device was based upon reading rate. The results are presented in 
Figure 3. The results demonstrated no difference in accuracy or reading speed with these two methods; the rate of Optacon reading agreed with estimates made by Goldish and Taylor (1974) of approximately 30 to 60 words/min. The mean reading rates with the Optacon system were 2.87 and 3.13 characters/sec with the interactive output device.

Of particular interest is the amount of experience the readers had had with each device: a minimum of 2 years with the Optacon system and a maximum of $15 \mathrm{~min}$ with the braille output device. Although the participants in this experiment were proficient braille readers and Optacon system users, the serial presentation of individual braille characters did not significantly degrade reading ability when compared with the more continuous display of characters experienced during Optacon reading. Both devices use serial presentation of information, but it should be noted that the Optacon presents letter shapes and contours to the reader that move in a continuous manner across the fingertip, whereas the electronic braille display unit translates ASCII text to braille code and then displays single braille characters sequentially at only one position.

One obvious advantage of the braille output device over a vibrotactile display is the negligible amount of practice needed to use the device. If the user is a braille reader, then the single prerequisite for its use has been satisfied, and an extended training period such as that required with a vibrotactile display is not needed. It is expected that increased experience with an interactive single electronic braille cell as an output device would improve performance. It may be that reading rates with this device will approach the reading speeds found with printed braille text. An increase in Optacon performance is not anticipated because of problems encountered in using a vibrotactile display. That is, Optacon reading speed is limited by lateral masking effects produced by tactile images

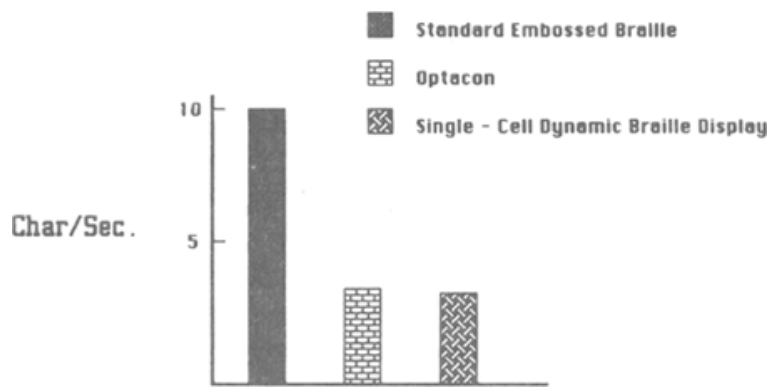

Figure 3. Reading rates as a function of method and device used.

moving across the fingertip and adaptation effects following extended use of the vibrotactile display (Craig, 1976).

Further development of the braille output device is expected to improve its performance. For example, reading rates could be improved by adding a variable-speed autorepeat function, which would allow the automatic presentation of characters to be scanned by a sustained keystroke. This study has demonstrated that the serial presentation of braille characters at a single position is a viable method of information transfer between computers and braille readers.

\section{REFERENCES}

Cralg, J. C. (1976). Vibrotactile letter recognition: The effects of a masking stimulus. Perception \& Psychophysics, 20, 317-326.

Goldish, L. H., \& TAYLOR, H. E. (1974). The Optacon: A valuable device for blind persons. The new outlook for the blind, 68, 49-56. TELESENSORY SySTEMS. (1971a). The Optacon: A print reading system for the blind. Palo Alto, CA: Author.

TELESENSORY SySTEMS. (1971b). The Versabraille system user's manual. Palo Alto, CA: Author.

TRIFORMATION SySTEMS. (1971). Microbrailler user's manual. Stuart, FL: Author 